NASA-CR-187,636

NASA Contractor Report 187636

ICASE Report No. 91-74

NASA-CR-187636

19920000953

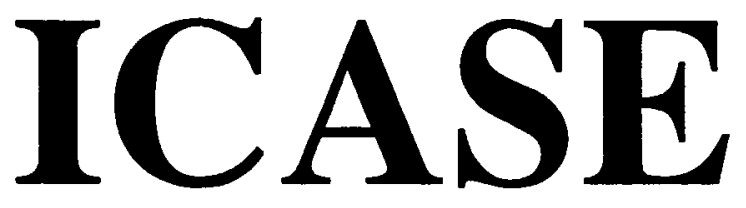

STABILITY OF THE FLOW AROUND A CYLINDER: THE SPIN-UP PROBLEM

S. R. Otto

Contract No. NAS1-18605

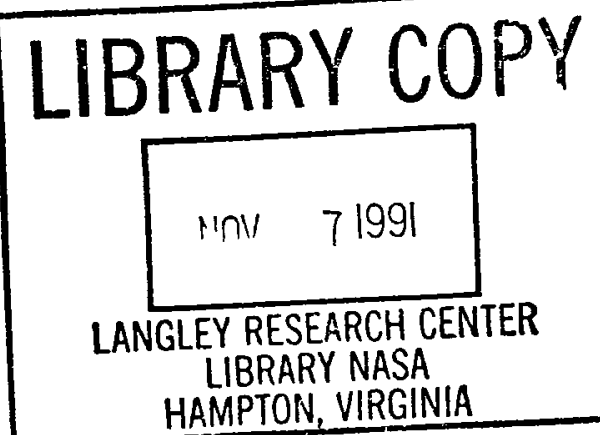

September 1991

Institute for Computer Applications in Science and Engineering NASA Langley Research Center

Hampton, Virginia 23665-5225

Operated by the Universities Space Research Association

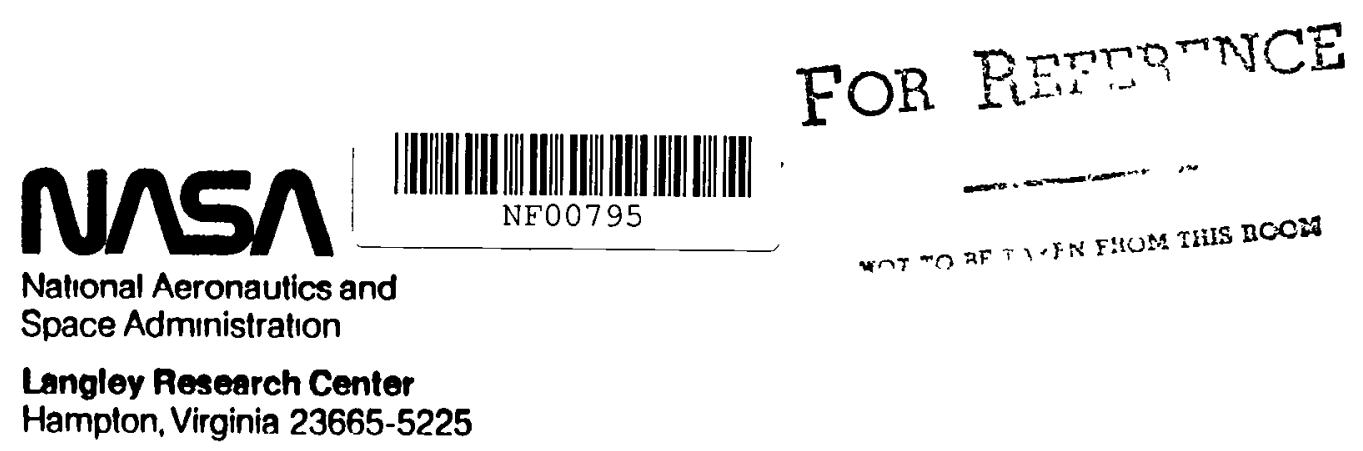




\title{
STABILITY OF THE FLOW AROUND A CYLINDER: THE SPIN-UP PROBLEM
}

\author{
S. R. Otto ${ }^{1}$ \\ Mathematics Department \\ Oxford Road \\ University of Manchester \\ Manchester \\ M13 9PL \\ U. K.
}

\begin{abstract}
Our concern is with the flow around an infinite cylinder, which at a certain instant is impulsively started to spin. The growth of vortices in the resulting boundary layer occurring outside the cylinder is investigated. This layer is essentially a Rayleigh layer which grows with time, so the mechanism involved is similar to that studied in Hall (1983). Vortices with wavenumber comparable to the layer thickness are shown to be described by partial differential equations. It is found necessary to solve the unsteady partial differential equations that govern the system numerically. We assume that the Rayleigh layer is thin so particles are confined to move in a path with radius of curvature the same as the cylinder. The Görtler number is a function of time, so we consider the time scale which produces an order one Görtler number. We consider the right hand branch calculation by letting the time tend to infinity, also inviscid Görtler modes are considered.
\end{abstract}

\footnotetext{
${ }^{1}$ This research was supported by the National Aeronautics and Space Adminstration under NASA Contract No NAS1-18605 whlle the author was in residence at the Institute for Computer Applications in Science and Engineering (ICASE), NASA Langley Research Center, Hampton, VA 23665
} 


\section{$\S 1 \quad$ Introduction}

The problem we are considering, is that of an infinite cylinder held fixed, until at a certain instant when a torsional impulsive velocity is imparted to the cylinder, without loss of generality this time may be considered to be $t=0$. The cylinder is immersed in a fluid of kinematic viscosity $\nu$ and density $\rho$, and the cylinder has radius $a$. The torsional velocity imparted to the cylinder is $\Omega$. It is known that a layer of thickness $a(\nu t)^{\frac{1}{2}}$ occurs at the cylinder, this is a Rayleigh layer which was shown to occur on a infinite flat plate by Stokes (1855) and later by Rayleigh (1911). We consider small time scales so that the resulting layer is thin and thus the particles contained in this layer can be considered to move in paths with the same radius of curvature as the cylinder. We may define the Görtler number as

$$
G=\frac{\Omega^{2} a^{2}(\nu t)^{\frac{3}{2}}}{a \nu^{2}}
$$

in the same way as in Seminara \& Hall (1976) and Hall (1984). These papers considered centrifugal instabilities in Stokes layers, so $(\nu t)^{\frac{1}{2}}$ is replaced by $(\nu / \omega)^{\frac{1}{2}}$, where $\omega$ is the frequency of the oscillations. The Reynolds number is defined here by

$$
R_{e}=\frac{\Omega a^{2}}{\nu} .
$$

The ensuing problem is similar initially to Hall (1983), who considered a spatially growing boundary layer occurring on a concave wall. We wish to investigate vortices similar to those iound to occur by Taylor (1923) and Görtler (1940). In Hall (1982 a,b) a self-consistent asymptotic description of the growth of short wavelength Görtler vortices was given. In particular in Hall (1982 a), it was shown that the equations governing the centrifugal instabilities are partial differential and not ordinary differential equations as discussed by Görtler (1940) and subsequent authors. In Hall (1982 b) the nonlinear development of the vortices was considered. In this paper we concentrate on disturbances with wavelengths initially of order one, but will consider the right hand branch in terms of local wavelength and local Görtler number. 
We may now apply the usual non-dimensionalisation and non-dimensionalise time with respect to $a^{2} / \nu$, thus the Görtler number in (1.1) may be written in terms of the Reynolds number $R_{e}$, and the non-dimensional time $T$ as

$$
G=R_{e}^{2} T^{\frac{3}{2}}
$$

To invoke the mechanism as described in Seminara \& Hall (1976), it is necessary to regard $G$ as order one, and this can be seen to occur if $T \sim R_{e}^{-\frac{4}{3}}$, and since we consider $R_{e} \gg 1$, this is a small time scale. We may define a similarity variable $\eta$, and thus it is possible to solve the basic flow equations in the resulting layer, note that we consider a radial scaling of $R_{e}^{-\frac{2}{3}}$ so that

$$
\xi=\frac{R-a}{2 T^{\frac{1}{2}}}=\mathrm{O}(1)
$$

where $R$ is a dimensional radial variable. The essential point here is that the equations governing the motion in this layer involve temporal derivatives as well as radial ones, this is equivalent to ensuring $x$-derivatives are retained in spatial problems, a fact that seems to have been first noted by Smith (1955). Smith (1955) retained some of the terms associated with the non-parallelism of the Görtler problem. For TollmienSchlichting waves Bouthier (1973) and Gaster (1974) incorporated extra terms to appreciate the effect of boundary layer growth, which greatly improved the comparison between experimental and theoretical results. Although it was noted by Gaster (1974) that the Bouthier-Gaster work just amounts to a successive approximation technique, Smith (1979) attempts to remedy this and does so effectively. Here we consider an unsteady instability, this is included in Kerczek \& Davis (1974) and subsequently in Kerczek \& Davis (1976) and Kerczek (1982), where in these papers they consider periodic flow. The description of the flow in a boundary layer growing with time on a semi-infinite flat plate was attempted in Otto (1992 b), employing a triple deck structure as in Smith (1979 a), so it is known theoretically that vortices occur in a time dependent growing boundary layer.

Here we concentrate on disturbances, of order one wavelengths as mentioned previously as these are most likely to be of practical importance. The development of 
the vortices is caused by the inclusion of a small hump. This hump is considered to be the same size as the boundary layer, this technique was employed in Denier, Hall, \& Seddougui (1991) in the spatially varying Görtler problem. Various forms of this hump are chosen and give rise to different regions of vortex activity, subsequently referred to as vortex wedges. In this paper we do not have the freedom of Denier, Hall \& Seddougui (1991) to introduce the disturbance at a certain spatial position as we have said that the impulsive motion starts at time equals zero, and the hump must be present for all time.

The identification of the time station $\bar{T}$, when a particular vortex wavenumber is neutral incurs the same problem as in non-parallel spatial calculations, refer to Hall (1983), Gaster (1974) and Smith (1979 a). In this paper the flow quantity chosen to be representative of the vortex size is

$$
E=\int_{0}^{\infty} \tilde{U}^{2} d Y,
$$

and thus the neutral position is defined by the position where $\sigma$, defined by

$$
\sigma=E^{-1} \frac{d E}{d T}
$$

becomes zero.

Thus at this time station we may calculate the local wavenumber $a_{\bar{T}}$ and the local Görtler number $G_{\mathcal{T}}$. The values of the equivalent pair in Denier, Hall, \& Seddougui (1991) are found to depend on the initial form of the disturbance. The initial form of the disturbance is a function of the hump at the wall.

We now go to investigate inviscid Görtler modes, where we have $a_{\bar{T}} \sim 1$ and $G_{\tilde{T}} \gg 1$ described in Denier, Hall, \& Seddougui (1991). We also consider the righthand branch problem such that for $a_{\bar{T}} \gg 1$, we have $G_{\bar{T}} \sim a_{\bar{T}}^{4}$, as investigated in Hall (1982 a). In this problem we have constant curvature, thus the right hand branch cannot be encountered by letting $\kappa \sim X^{\frac{1}{2}}$ as in Hall (1982 a). In order to investigate the right 
hand branch it is found necessary to consider very small initial wavelengths $k$ at a time $T$ such that,

$$
k \sim T^{-\frac{1}{8}} \quad \text { with } \quad T \gg 1 .
$$

We also consider matching between the inviscid solutions and right hand branch solutions as in Denier, Hall \& Seddougui (1991).

The approach adopted in the remainder of this paper is as follows; in section 2 we derive the linear $\mathrm{O}(1)$ wavenumber regime problem, in section 3 the inviscid Görtler problem is tackled, section 4 contains details of the right hand branch calculations, section 5 includes description of the numerical techniques involved in the solution of the equations derived in sections 3 and 4 . Finally section 6 contains results and discussions including comments on matching between the solutions of section 4 and section 5, and a discussion of possible further developments to take account of fully nonlinear right hand branch calculations. A discussion of extension to the problem to further geometries is also included. 


\section{$\S 2$ Formulation of linear order one problem}

We non-dimensionalise the velocity field with respect to $a \Omega$, and the pressure terms with respect to $\rho a^{2} \Omega^{2}$. The induced motion is taken to be independent of $\theta$, the coordinate system employed is that of cylindrical polars with components $(r, \theta, z)$, and corresponding velocity components $(u, v, w)$, the Navier-Stokes equations then become,

$$
\begin{gathered}
\frac{1}{R_{e}} \frac{\partial U}{\partial t}+U \frac{\partial U}{\partial r}+W \frac{\partial U}{\partial z}-\frac{V^{2}}{r}=-\frac{\partial P}{\partial r}+\frac{1}{R_{e}}\left(\left(\frac{\partial^{2}}{\partial r^{2}}+\frac{\partial^{2}}{\partial z^{2}}\right)+\frac{1}{r} \frac{\partial}{\partial r}-\frac{1}{r^{2}}\right) U \\
\frac{1}{R_{e}} \frac{\partial V}{\partial t}+U \frac{\partial V}{\partial r}+W \frac{\partial V}{\partial z}+\frac{V U}{r}=\frac{1}{R_{e}}\left(\left(\frac{\partial^{2}}{\partial r^{2}}+\frac{\partial^{2}}{\partial z^{2}}\right)+\frac{1}{r} \frac{\partial}{\partial r}-\frac{1}{r^{2}}\right) V \\
\frac{1}{R_{e}} \frac{\partial W}{\partial t}+U \frac{\partial W}{\partial r}+W \frac{\partial W}{\partial z}=-\frac{\partial P}{\partial z}+\frac{1}{R_{e}}\left(\left(\frac{\partial^{2}}{\partial r^{2}}+\frac{\partial^{2}}{\partial z^{2}}\right)+\frac{1}{r} \frac{\partial}{\partial r}\right) W \\
\frac{\partial U}{\partial r}+\frac{U}{r}+\frac{\partial W}{\partial z}=0 .
\end{gathered}
$$

We now introduce an axi-symmetric hump at the cylinder, defined by $r=1+\delta f(z)$ . Following the arguments in the introduction, it is found necessary to look at a time scaling $T \sim R_{e}^{-\frac{4}{3}}$ so that the Görtler number is order one, and in this section it will be taken to be one with no loss of generality. Thus we define boundary layer variables, $\eta$ and $Z$ given by

$$
r=1+R_{e}^{-\frac{2}{3}} \eta, \quad z=R_{e}^{-\frac{2}{3}} Z,
$$

and a time boundary layer variable $\tau$, defined by

$$
T=R_{e}^{-\frac{4}{3}} \tau
$$

To determine the basic flow we assume it to be independent of $Z$ and a function of $\xi$, a similarity variable given by

$$
\xi=\frac{\eta}{2 \tau^{\frac{1}{2}}}
$$

The basic flow is found to be governed by

$$
\frac{\partial^{2} \bar{v}}{\partial \xi^{2}}+2 \xi \frac{\partial \bar{v}}{\partial \xi}=0
$$


with boundary conditions

$$
\begin{array}{ccc}
\bar{v}=0 & \text { as } & \xi \rightarrow \infty \\
\bar{v}=1 \quad \text { at } & \xi=0
\end{array}
$$

The solution of (2.4) is the error function, given by

$$
\bar{v}(\xi)=\frac{2}{\sqrt{\pi}} \int_{\xi}^{\infty} e^{-\hat{\xi}^{2}} d \hat{\xi},
$$

We now introduce a disturbance so that the flow field is now given by

$$
\mathbf{u}=(0, \bar{v}, 0, \bar{p})+\delta\left(R_{e}^{-\frac{1}{3}} \tilde{u}, \tilde{v}, R_{e}^{-\frac{1}{3}} \tilde{w}, R_{e}^{-\frac{2}{3}} \tilde{p}\right)+\cdots
$$

where the quantities with tildes are functions of $\eta, Z$ and $\tau$, and, as previously stated independent of $\theta$. We take $\delta$ to be a small vanishing parameter, so that we are in a linear regime, with this assumption the governing equations are found to be,

$$
\begin{gathered}
\frac{\partial \tilde{u}}{\partial \tau}-2 \bar{v} \tilde{v}=-\frac{\partial \tilde{p}}{\partial \eta}+\left(\frac{\partial^{2}}{\partial \eta^{2}}+\frac{\partial^{2}}{\partial Z^{2}}\right) \tilde{u} \\
\frac{\partial \tilde{v}}{\partial \tau}+\frac{\partial \bar{v}}{\partial \eta} \tilde{u}=\left(\frac{\partial^{2}}{\partial \eta^{2}}+\frac{\partial^{2}}{\partial Z^{2}}\right) \tilde{v} \\
\frac{\partial \tilde{w}}{\partial \tau}=-\frac{\partial \tilde{p}}{\partial Z}+\left(\frac{\partial^{2}}{\partial \eta^{2}}+\frac{\partial^{2}}{\partial Z^{2}}\right) \tilde{w} \\
\frac{\partial \tilde{u}}{\partial \eta}+\frac{\partial \tilde{w}}{\partial Z}=0
\end{gathered}
$$

with boundary conditions given by

$$
\tilde{u}=\tilde{v}=\tilde{w}=0 \quad \text { as } \quad \eta \rightarrow \infty,
$$

so that the disturbance is confined to the Rayleigh layer,

$$
\tilde{u}=\tilde{v}=\frac{\partial \tilde{v}}{\partial \eta}=0 \quad \text { at } \quad \eta=f(Z) .
$$

Here $(2.7 \mathrm{f})$ represents the no-slip boundary conditions at the hump on the cylinder, which is chosen to be scaled with the boundary layer thickness multiplied by the disturbance size $\delta$. Note that since we looking close to the cylinder the terms proportional 
to secondary radial corrections are neglected, such as $1 / r \frac{\partial}{\partial r}$. Let us now apply the Fourier transform to the disturbance quantities, thus the transform $\tilde{U}$ of $\tilde{u}$ is defined by

$$
\tilde{U}=\frac{1}{\sqrt{2 \pi}} \int_{-\infty}^{\infty} \tilde{u} e^{i k Z} d Z .
$$

Thus the system (2.7) now becomes

$$
\begin{gathered}
\frac{\partial \tilde{U}}{\partial \tau}-2 \bar{v} \tilde{V}=-\frac{\partial \tilde{P}}{\partial \eta}+\left(\frac{\partial^{2}}{\partial \eta^{2}}-k^{2}\right) \tilde{U} \\
\frac{\partial \tilde{V}}{\partial \tau}+\frac{\partial \bar{v}}{\partial \eta} \tilde{U}=+\left(\frac{\partial^{2}}{\partial \eta^{2}}-k^{2}\right) \tilde{V}, \\
\frac{\partial \tilde{W}}{\partial \tau}=-i k \tilde{P}+\left(\frac{\partial^{2}}{\partial \eta^{2}}-k^{2}\right) \tilde{W}, \\
\frac{\partial \tilde{U}}{\partial \eta}+i k \tilde{W}=0,
\end{gathered}
$$

with boundary conditions of

$$
\begin{gathered}
\tilde{U}=\tilde{V}=\tilde{W}=0 \quad \text { as } \quad \eta \rightarrow \infty, \\
\tilde{U}=\tilde{W}=0, \quad \tilde{V}=-F \frac{\partial \bar{v}}{\partial \eta} \quad \text { at } \quad \eta=0 .
\end{gathered}
$$

where $F$ is the Fourier transform of the hump $f(z)$. Note that is the absence of the hump there would be no vortex mechanism. Now by elementary manipulation, we may eliminate $\tilde{W}$ and $\tilde{P}$, to obtain the coupled partial differential system,

$$
\begin{gathered}
\left(\frac{\partial^{2}}{\partial \eta^{2}}-k^{2}-\frac{\partial}{\partial \tau}\right)\left(\frac{\partial^{2}}{\partial \eta^{2}}-k^{2}\right) \tilde{U}=2 k^{2} \tilde{v} \tilde{V}, \\
\left(\frac{\partial^{2}}{\partial \eta^{2}}-k^{2}-\frac{\partial}{\partial \tau}\right) \tilde{V}=\frac{\partial \bar{v}}{\partial \eta} \tilde{U}
\end{gathered}
$$

this system is the Görtler equations for a vortex with temporal growth, with Görtler number equal to one. It is now noted that there will be a discontinuity in the disturbance flow field due to the impulsive start, so it is necessary to solve the problem asymptotically for small $\tau$. Since the solution of (2.10) necessitates a numerical approach, this asymptotic solution may be used as an initial condition. It can be seen 
from $(2.10 \mathrm{~b})$ that $\tilde{V}(0, \tau) \sim \tau^{-\frac{1}{2}}, \tilde{U}(0, \tau) \sim \tau^{\frac{3}{2}}$ for $\tau \ll 1$ following this scaling we seek a solution of the form

$$
\begin{gathered}
\tilde{V}=\tau^{-\frac{1}{2}} \tilde{V}_{0}(\xi)+\cdots \\
\tilde{U}=\tau^{\frac{3}{2}} \tilde{U}_{0}(\xi)+\cdots
\end{gathered}
$$

where $\xi$ is the similarity variable defined by $(2.3)$, it may be shown that $\tilde{V}_{0}(\xi)$ satisfies

$$
\frac{\partial^{2} \tilde{V}_{0}}{\partial \xi^{2}}+2 \xi \frac{\partial \tilde{V}_{0}}{\partial \xi}+2 \tilde{V}_{0}=0
$$

with boundary conditions

$$
\tilde{V}_{0}(0)=2 \frac{F(k)}{\sqrt{\pi}} \quad \text { and } \quad \tilde{V}_{0}(\infty)=0
$$

where $F(k)$ is the Fourier transform of $f(z)$, the form of the hump. The solution of (2.12) is given by

$$
\tilde{V}_{0}=2 \frac{F(k)}{\sqrt{\pi}} e^{-\xi^{2}}
$$

Note that we take the radial component $\tilde{U}_{0}$ to be zero for small $\tau$, as it is $Q\left(\tau^{2}\right)$ smaller than $\tilde{V}_{0}$. The equations may now be solved numerically, the solution is discussed in section 5. Note that since this system is linear $F(k)$ may be taken to be unity, this corresponds to a $\delta$-function shaped hump, so is relevant to a localised imperfection on the surface of the cylinder.

\section{§3 The inviscid limit of the Görtler problem}

In Denier, Hall \& Seddougui (1991) the inviscid Görtler problem was discussed, it corresponds to a large Görtler number and order one wavenumbers. The temporal inviscid Görtler problem is virtually identical to the inviscid problem set as an exercise by Drazin \& Reid (1979) and solved by Floryan (1986). Thus we have the governing equations (2.10) with a Görtler number other than one, taken to be $\tilde{G}$

$$
\left(\frac{\partial^{2}}{\partial \eta^{2}}-k^{2}-\frac{\partial}{\partial \tau}\right)\left(\frac{\partial^{2}}{\partial \eta^{2}}-k^{2}\right) \tilde{U}=2 \tilde{G} k^{2} \bar{v} \tilde{V}
$$




$$
\left(\frac{\partial^{2}}{\partial \eta^{2}}-k^{2}-\frac{\partial}{\partial \tau}\right) \tilde{V}=\frac{\partial \bar{v}}{\partial \eta} \tilde{U}
$$

We now consider a solution of (3.1) in the inviscid regime, that is $\tilde{G} \gg 1$ with $k=O(1)$. It may be shown that the growth rate is proportional to $\tilde{G}^{\frac{1}{2}}$ following Denier, Hall \& Seddougui (1991). Thus the disturbance in this regime takes the form

$$
(\tilde{U}, \tilde{V})=E\left(U, \tilde{G}^{-\frac{1}{2}} V\right)
$$

where

$$
E=\exp \left(\tilde{G}^{\frac{1}{2}} \int^{\tau} \beta+\cdots\right),
$$

This form of the disturbance may be substituted into (3.1), and the two equations can be combined to produce,

$$
\beta^{2}\left(\frac{\partial^{2}}{\partial \eta^{2}}-k^{2}\right) U-2 k^{2} U \bar{v} \frac{\partial \bar{v}}{\partial \eta}=0
$$

In Denier, Hall \& Seddougui (1991) the equivalent equation has a closed form of solution. Unfortunately (3.3) does not, so thus must be solved numerically. The equation (3.3) was solved using a fourth order Runge-Kutta scheme. The growth rate is plotted against $k$ in figure 1. It is interesting to look at the solution of (3.3) as $k$ increases to infinity, thus effectively encountering the right hand branch. We anticipate that the growth rate tends to a constant $\beta_{0}^{\frac{1}{2}}$, so that we expand the growth rate as

$$
\beta=\left(\beta_{0}+k^{-\frac{2}{3}} \beta_{1}+\cdots\right)^{\frac{1}{2}}
$$

the size of the second order term is found from the size of the layer found to occur adajacent to $\eta=0$, from elementary observations of the numerical results.

Thus we introduce a boundary layer variable $\chi$, given by

$$
\eta=k^{-\frac{2}{3}} \chi
$$

In this layer it is known that the basic flow $\bar{v}$ tends to

$$
1-\lambda k^{-\frac{2}{3}} \chi+\cdots,
$$


where $\lambda=-\left.\bar{v}_{\eta}\right|_{\eta=0}=\frac{2}{\sqrt{\pi}}$. Expand the disturbance $U$ as

$$
U=U_{0}+k^{-\frac{2}{3}} U_{1}+\cdots,
$$

at leading order we obtain

$$
-\beta_{0} U_{0}+2 \lambda U_{0}=0
$$

This is a compatibility condition, so for non-trivial solutions we require $\beta_{0}=2 \lambda$, at next order we obtain

$$
\frac{d^{2} U_{0}}{d \chi^{2}}-\left(\lambda\left(\chi+\frac{\beta_{1}}{\beta_{0} \lambda}\right)\right) U_{0}=0 .
$$

For decaying solutions as $\chi \rightarrow \infty$, the solution of (3.9) is given by

$$
U_{0}=K \mathrm{Ai}\left[\lambda^{\frac{1}{3}}\left(\chi+\frac{\beta_{1}}{\beta_{0} \lambda}\right)\right] .
$$

To solve the boundary condition at $\chi=0$ we require that

$$
\operatorname{Ai}\left(\frac{\beta_{1}}{\beta_{0} \lambda^{\frac{2}{3}}}\right)=0
$$

thus we can find the second order growth rate $\beta_{1}$ in terms of the eigenvalues of the Airy function. Notice that this function has many zeros on the negative real axis, we are interested in the highest value of the growth rate, that is the first eigenvalue. Thus as $k \rightarrow \infty$, we have

$$
\beta \rightarrow \sqrt{2 \lambda}-\frac{3.372}{3^{\frac{1}{3}}} \lambda^{\frac{5}{3}} k^{-\frac{2}{3}}+\cdots,
$$

which we compare with the right hand branch growth rate in section 4 , this result is also included in figure 1 , note this agreement is reasonable, but it should be noted that $k^{-\frac{2}{3}}$ term does not tend to zero very quickly. Also included in figure 1 is the righthand growth rate value, and it can be seen that the solid curve is tending to this value.

\section{$\S 4$ Right hand branch calculation}

We consider the right hand branch where $k$ is large, and large Görtler number, $\tilde{G}$ as introduced in section 3 . Neutral vortices in this regime were described by Hall (1982 a). In the neighbourhood of the neutral position, temporally amplified modes 
with growth rate $O\left(k^{2}\right)$, are found to occur. In this regime, we know for neutral modes $\tilde{G} \sim k^{4}$, so we write

$$
k=\lambda^{\dagger} \tilde{G}^{\frac{1}{4}}
$$

We note that the vortex activity is likely to be concentrated where the Rayleigh criterion is most violated, for the problem considered here this occurs at the wall, which is the same as the Taylor problem, one of the problems considered by Hall (1982 a). We consider a disturbance proportional to

$$
E=\exp \left(\frac{i z}{\epsilon}+\frac{1}{\epsilon^{2}} \int^{t} \beta_{0}(\phi)+\epsilon \beta_{1}(\phi)+\cdots d \phi\right)
$$

where $1 / \epsilon=k$, and thus $\tilde{G}$ may be expanded as

$$
\tilde{G}=\sum_{n=0}^{\infty} g_{n} \epsilon^{-4+n} .
$$

Thus the governing equations become

$$
\begin{gathered}
\left(\epsilon^{2} \frac{\partial^{2}}{\partial \eta^{2}}-1-\epsilon^{2} \frac{\partial}{\partial \tau}\right)\left(\epsilon^{2} \frac{\partial^{2}}{\partial \eta^{2}}-1\right) U=2 \epsilon^{2} \tilde{G} \bar{v} V, \\
\left(\epsilon^{2} \frac{\partial^{2}}{\partial \eta^{2}}-1-\epsilon^{2} \frac{\partial}{\partial \tau}\right) V=U \frac{\partial \bar{v}}{\partial \eta}
\end{gathered}
$$

Here we consider non-neutral solutions, thus $\beta_{0}$ is non-zero it is possible to derive a compatibility relation, so in (4.4) we now have

$$
(U, V)=\left(u_{0}, k^{-2} v_{0}\right) E
$$

We introduce a layer of thickness $\lambda^{\dagger^{-\frac{1}{2}}} \tilde{G}^{-\frac{1}{8}}$, that is $k^{-\frac{1}{2}}$. So substituting (4.5) into (4.4) in this layer, located at the cylinder, we obtain

$$
\begin{aligned}
& \left(\lambda^{\dagger^{2}}+\beta\right) \lambda^{\dagger^{2}} u_{0}=2 v_{0}, \\
& \left(\lambda^{\dagger^{2}}+\beta\right) v_{0}=\lambda u_{0} \lambda^{\dagger^{2}}
\end{aligned}
$$

where we have assumed for $\eta \ll 1, \bar{v} \rightarrow 1-\lambda \eta+\cdots$. Thus combining (4.6 a) and (4.6 b) we obtain,

$$
\left(\lambda^{\dagger^{2}}+\beta\right)-2 \lambda=0
$$


Thus as $\lambda^{\dagger} \rightarrow 0$, the growth rate $\beta$ tends to $\sqrt{2 \lambda}$, which agrees with the inviscid problem as $k \rightarrow \infty$. Note that we also have an order one cut off point where the growth rate is zero given by

$$
\lambda^{\dagger}=(2 \lambda)^{\frac{1}{4}}
$$

Thus it is now possible to continue in the same manner as Hall (1982 a) and derive the form of the neutral modes, this analysis is not included as the conversion to this case is trivial and has the same conclusions. Note that in Denier, Hall \& Seddougui (1991) they found that the position of the vortex activity varies with $\lambda^{\dagger}$, we do not have that here because our growth rate is temporal and associated with $\partial / \partial \tau$, whereas in Denier, Hall \& Seddougui (1991) the growth rate is associated with $\bar{u} \partial / \partial x$. They also found as $\lambda^{\dagger} \rightarrow 0$, that there is a singularity in the growth rate and thus there is an intermediate $G^{\frac{1}{3}}$ wavenumber regime required to match with the inviscid problem but that does not occur here, as we have direct correlation between large- $k$ inviscid growth rate and small- $k$ right hand branch growth rate.

\section{$\S 5$ The numerical work}

Now let us discuss the numerical scheme employed to integrate the inhomogeneous system for $\tilde{U}$ and $\tilde{V}$. The scheme is based on a finite difference scheme in the $\eta$-direction together with an Euler step in $\tau$. Thus for example (2.10 b) becomes

$$
\frac{\tilde{V}_{i}^{+}}{\Delta \tau}-\left(\frac{\tilde{V}_{i+1}^{+}-2 \tilde{V}_{i}^{+}+\tilde{V}_{i-1}^{+}}{h^{2}}\right)=\bar{v}_{i}^{-}\left(\frac{\tilde{V}_{i+1}^{-}-\tilde{V}_{i-1}^{-}}{2 h}\right)-k^{2} \tilde{V}_{i}^{-}+\frac{\tilde{V}_{i}^{-}}{\Delta \tau},
$$

where $i$ denotes grid position in the $\eta$-direction, whilst \pm denotes the function evaluated at $\tau+\Delta \tau$ and $\tau$ respectively. Furthermore $h$ and $\Delta \tau$ are the step lengths in the $\eta$ and $\tau$ directions respectively. If the disturbance velocity field is known at time $\tau$, then the tridiagonal system associated with (2.1) can be solved to give $\tilde{V}$ at time $\tau+\Delta \tau$, a similar discretization of $(2.10 \mathrm{a})$ then produces a pentadiagonal system for $\tilde{U}$, this system can be solved for $\tilde{U}$ at time $\tau+\Delta \tau$; this procedure can then be repeated to march forward in time. This scheme is fully implicit so is stable for $\Delta \tau=O(h)$ rather than $\Delta \tau=O\left(h^{2}\right)$ which would be the case with an explicit scheme. 
After some grid checks we found that 300 steps in $\eta$, with infinity $\eta=20$ was sufficient to present the given results to sufficient accuracy. The value of $\Delta \tau$ used to calculate the results given, was $\Delta \tau=0.001$. The initial value of $\tau$ where the calculations were begun was varied until it was sufficient small to calculate $\tilde{U}$ and $\tilde{V}$ with sufficient accuracy, the value used was $\Delta \tau=0.01$.

As an indication of vortex growth, the energy was computed, defined by

$$
E=\int_{0}^{\infty} \tilde{U}^{2} d \eta,
$$

as mentioned in the introduction and then the growth rate $\beta$ was calculated, defined by

$$
\beta=\frac{d E}{d \tau} E^{-1} .
$$

For all our calculations $\beta$ was found initially to be negative so at sufficient value of time the flow is stable. At a later time, say $\tau_{0}$, we found that $\beta$ becomes positive until $\tau_{1}$, where $\beta$ again becomes negative. Thus there is a finite time interval where the basic unsteady boundary layer is unstable. At $\tau=\tau_{0}$ and $\tau=\tau_{1}$, the instantaneous wavenumber $k \tau^{\frac{1}{2}}$ can be plotted against the instantaneous Görtler number $\tilde{G} \tau^{\frac{3}{2}}$ to generate the neutral curve for spin-up, this is shown in figure 2 , for $\tilde{G}=1$.

We conclude that spin-up is instantaneously unstable for small time interval, $\sim R_{e}^{-\frac{4}{3}}$; during this time turbulent flow will almost certainly be set up so that stable rigid body rotation may never be achieved in the practical situation. Furthermore we note that the induced velocity for any shaped bump can be found by numerical integration of the transformed velocity field. Several different humps were tried, and the results were found to be quantitatively similar, so we only include the plots for the humps included in Denier, Hall \& Seddougui (1991). In the graphs the ordinate is the $z$-axis and the abscissa represents the time axis. Figure 3, represents the contours of maximum $V$ component for the hump $\pi^{\frac{1}{2}} / 4 e^{-z^{2} / 16}$, and figure 4 the contours.for $\pi^{\frac{1}{2}} z / 32 e^{-z^{2} / 16}$. Note these are basically the symmetric and anti-symmetric cases. The region of vortex activity is basically the same for any form of hump, even step functions. 


\section{$\S 6$ Conclusion}

We have shown for the spin-up problem, that Görtler vortices may occur when forced by an axi-symmetric hump at a cylinder started to move impulsively, also that the shape of the hump does not effect the amplitude of the vortices found to evolve, or greatly alter the time of the onset of the instability.

We note that the growth rate, for an order one initial wavelength has two zeros, namely $\tau=\tau_{0}$ and $\tau=\tau_{1}$, thus between $\tau_{0}$ and $\tau_{1}, \beta$ the growth rate is positive and during this finite time interval spin up flow is unstable. The neutral curve is plotted for the humps, notice it is independent of hump shape as it is done before the Fourier inversion. We also produce figures showing vortex growth for each hump considered, the plots are of time against the maximum value of $V$ for a given $z$, this quantity is taken to be representative of the vortex amplitude. Note that to graphical accuracy the wavelength of the vortices obtained corresponds to the critical wavelength from the relevant neutral curve.

In section 3, we described the inviscid Görtler modes, which we found to have a growth rate of $\sqrt{2 \lambda} \tilde{G}^{\frac{1}{2}}$, as $k \rightarrow \infty$, in section 4 the growth rate of the right hand branch in the limit $k \rightarrow 0$, was found to tend to $\tilde{G}^{\frac{1}{2}} \sqrt{2 \lambda}$, thus we have matching of the growth rates, there is therefore no further structure brought on by a singularity in the growth rate, as was found to occur in Denier, Hall, \& Seddougui (1991).

In section 4 we did not include the description of neutral vortices but briefly we see that the first order disturbance term satisfies,

$$
\frac{\partial^{2} V_{0}}{\partial \theta^{2}}-\frac{\theta^{2}}{4} V_{0}-a V_{0}=0
$$

where

$$
\theta=\tau^{\frac{1}{4}} \chi\left(-4\left(\frac{V_{02}^{+}}{V_{01}^{+}}+\frac{V_{03}^{+}}{V_{01}^{+}}\right)\right)^{\frac{1}{4}}
$$

and

$$
a=\frac{V_{00}^{+} V_{01}^{+}}{3} \frac{g_{2}}{\alpha^{2}} \quad \text { where } \quad \alpha=\left(-4\left(\frac{V_{02}^{+}}{V_{01}^{+}}+\frac{V_{03}^{+}}{V_{01}^{+}}\right)\right)^{\frac{1}{4}}
$$


where the superscript + denotes evaluation at $\eta^{+}$, the position of vortex activity, in this case $\eta^{+}$equals zero. Thus $V_{0}$ is given by a parabolic cylinder function, ensuring the required decay as $\chi \rightarrow \infty$, if $a=-\frac{1}{2}-m$, thus we have

$$
g_{0}=-\frac{\tau^{\frac{1}{2}}}{V_{00}^{+} V_{01}^{+}} \quad g_{1}=0 \quad g_{2}=-\frac{3 \alpha^{2}}{2 V_{00}^{+} V_{01}^{+}},
$$

note the terms $V_{0 i}^{+}$are the coefficients of the Taylor series of the basic flow evaluated in a layer of thickness $k^{-\frac{1}{2}}$, situated at $\eta^{+}$.

It is also possible to extend the small wavelength analysis to a fully non-linear calculation, performing a similar calculation to Hall \& Lakin (1988).

It should be also noted that we may extend this analysis to further geometries, the first obvious extension would be to the case involving a sphere, a brief outline of the method involved follows. Firstly it is necessary to invoke slightly different scaling for a sphere involved in spin-up about an axis of symmetry, the scaling are now

$$
w \sim W, \quad u, v \sim a W^{2} / \nu, \quad r \sim a, \quad t \sim \nu / a^{2} \quad p \sim \rho W^{2} .
$$

These scaling are the same as those employed in the torsional case of Otto (1992 a), where $\omega$ the frequency is now replaced with $\nu / a^{2}$ Thus the governing equations become

$$
\begin{gathered}
\frac{\partial u}{\partial t}+R_{e}^{2}\left(u \frac{\partial u}{\partial r}+\frac{v}{r} \frac{\partial u}{\partial \theta}-\frac{v^{2}}{r}\right)-\frac{w^{2}}{r}=-\frac{\partial p}{\partial r}+\left(\nabla^{2} u-\frac{2 u}{r^{2}}-\frac{2}{r^{2}} \sin \theta \frac{\partial}{\partial \theta}(v \sin \theta)\right) \\
\frac{\partial v}{\partial t}+R_{e}^{2}\left(u \frac{\partial v}{\partial r}+\frac{v}{r} \frac{\partial v}{\partial \theta}-\frac{u v}{r}\right)-\frac{w^{2}}{r} \cot \theta=-\frac{1}{r} \frac{\partial p}{\partial \theta}+\left(\nabla^{2} v+\frac{2}{r^{2}} \frac{\partial u}{\partial \theta}-\frac{v}{r^{2} \sin \theta}\right) \\
\frac{\partial w}{\partial t}+R_{e}^{2}\left(u \frac{\partial w}{\partial r}+\frac{v}{r} \frac{\partial w}{\partial \theta}+\frac{u w}{r}+\frac{v w}{r} \cot \theta\right)=\left(\nabla^{2} w-\frac{w}{r^{2} \sin ^{2} \theta}\right) \\
\frac{1}{r^{2}} \frac{\partial}{\partial r}\left(r^{2} u\right)+\frac{1}{r \sin \theta} \frac{\partial}{\partial \theta}(v \sin \theta)=0
\end{gathered}
$$

where,

$$
\nabla^{2}=\frac{1}{r^{2}} \frac{\partial}{\partial r}\left(r^{2} \frac{\partial}{\partial r}\right)+\frac{1}{r^{2} \sin \theta} \frac{\partial}{\partial \theta}\left(\sin \theta \frac{\partial}{\partial \theta}\right)
$$


Note that we have assumed that the flow is independent of the azimuthal coordinate $\phi$, as was done in the torsional case of Otto (1992 a), this also corresponds to assuming the flow in the cylinderical case is independent of $\theta$. A Reynolds number has also been introduced, where this is given by $W a / \nu$.

From similar arguments to those employed in the torsional case discussed in Otto (1992 a), the onset of a vortex stability is most likely to occur at the equator, thus we regard the basic velocity in this area. The derivation of the basic flow was attempted in Benton (1965), and he showed the basic flow components to be given by,

$$
\begin{gathered}
\bar{w} \sim \sin \theta f(r, t), \\
\bar{v} \sim \cos \theta \sin \theta g(r, t) .
\end{gathered}
$$

Note in the region of $\pi / 2$ we introduce a boundary layer variable $\tilde{\theta} \ll 1$, and the basic flow quantities expand as

$$
\begin{gathered}
\bar{w} \sim f(r, t)+O\left(\tilde{\theta}^{2}\right) \ldots, \\
\bar{u} \sim O(\tilde{\theta})+\ldots .
\end{gathered}
$$

The Görtler number is given by

$$
G=R_{e}^{2} T^{\frac{3}{2}}
$$

and so in a similar manner to the cylinder problem, we employ a time scaling so that the Görtler number remains of order one, thus we introduce

$$
r=1+R_{e}^{-\frac{2}{3}} \eta, \quad t=R_{e}^{-\frac{4}{3}} \tau, \quad \theta=\frac{\pi}{2}+R_{e}^{-\frac{2}{3}} \tilde{\theta} .
$$

Now in the region of the equator of the sphere the basic flow is found to be given by an error function, we perturb this basic state by the quantity

$$
\left(R_{e}^{-\frac{4}{3}} \tilde{u}, R_{e}^{-\frac{4}{3}} \tilde{v}, R_{e}^{-\frac{2}{3}} \tilde{w}\right)
$$

As thus we arrive at the governing equations

$$
\frac{\partial \tilde{u}}{\partial \tau}-\bar{w} \tilde{w}=-\frac{\partial \tilde{p}}{\partial \eta}+\left(\frac{\partial^{2}}{\partial \eta^{2}}+\frac{\partial^{2}}{\partial \tilde{\theta}^{2}}\right) \tilde{u}
$$




$$
\begin{gathered}
\frac{\partial \tilde{v}}{\partial \tau}=-\frac{\partial \tilde{p}}{\partial \tilde{\theta}}+\left(\frac{\partial^{2}}{\partial \eta^{2}}+\frac{\partial^{2}}{\partial \tilde{\theta}^{2}}\right) \tilde{v} \\
\frac{\partial \tilde{w}}{\partial \tau}+\tilde{u} \frac{\partial \bar{w}}{\partial \eta}=\left(\frac{\partial^{2}}{\partial \eta^{2}}+\frac{\partial^{2}}{\partial \tilde{\theta}^{2}}\right) \tilde{w} \\
\frac{\partial \tilde{u}}{\partial \eta}+\frac{\partial \tilde{v}}{\partial \tilde{\theta}}=0
\end{gathered}
$$

Thus note if a Fourier transform is now applied to the these equations we arrive at the equations (2.9), with $\bar{v}$ replaced by $\bar{w}$. Therefore we have shown that a sphere involved in spin-up is locally unstable to the same vortex structure as that found at a cylinder in the same situation. Although it should be noted that as in the torsional case of Otto (1992 a), difficulties may be experienced actually at the equator, so extension of the problem into the complex $\theta$-plane may be necessary.

The author acknowledges Professor Philip Hall for suggesting this problem, and his assistance with the numerical calculations involved. The author wishes to thank Dr. A. P. Bassom for useful discussions, relevant to the inviscid modes. This work was done while the author was in receipt of a SERC research studentship. 


\section{References}

Benton, E. R. (1965) Laminar boundary layer on an impulsively started sphere. J. Fluid Mech. 23611

Bouthier, M. (1973) Stabilité linéare des ecoulements presque parallèles. Partie II. La couche limite de Blasius J. Méc (Fr.) 1275

Denier, J.P., Hall, P. \& Seddougui, S. (1991) On the receptivity problem for Görtler vortices: vortex motion induced by wall roughness Phil. Trans. Roy. Soc. A 33551

Drazin, P.G. \& Reid, W.H. (1979) Hydrodynamic Instability, Cambridge University Press

Floryan, J.M. (1986) Görtler instability of boundary layers over concave and convex walls Phys. Fluids 292380

Gaster, M. (1974) On the effects of boundary layer growth on flow stability $J$. Fluid Mech. 66465

Görtler, H. (1940) On the three dimensional instability of laminar boundary layers on concave walls NACA Tech. Memo no. 1375

Hall, P. (1982 a) Taylor-Görtler vortices in fully developed or boundary layer flows J. Fluid Mech. 124475

Hall, P. (1982 b) Centrifugal instabilities of circumferential flows in finite cylinders: the wide gap problem Proc. $R$. Soc. Lond. A 384389

Hall, P. (1983) The linear development of Görtler vortices in growing boundary layers J. Fluid Mech. 13041

Hall, P. \& Lakin, W.D. (1988) The fully non-linear development of Görtler vortices in growing boundary layers Proc. R. Soc. Lond. A 415421

Kerczek, C.H. von (1982) The instability of oscillatory plane Poiseuille flow J. Fluid Mech. 11691

Kerczek, C. von \& Davis, S.H. (1974) Linear stability of oscillatory Stokes layers J. Fluid Mech. 62753 
Kerczek, C. von \& Davis, S.H. (1976) The instability of a stratified periodic boundary layer J. Fluid Mech. 75287

Otto, S. R. (1992 a) On the instability of the flow around an oscillating sphere submitted to J. Fluid Mech.

Otto, S. R. (1992 b) A study of instabilities in a time dependent boundary layer. submitted to QJMAM

Rayleigh, Lord (1911) On the motion of solid bodies through a viscous liquid Phil. Mag. 21697

Seminara, G. \& Hall, P. (1976) Centrifugal instability of a Stokes layer: linear theory Proc. R. Soc. Lond. A 35029

Smith, A.M.O (1955) On the growth of Taylor-Görtler vortices along highly curved concave walls $Q$. Appl. Math. 13233

Smith, F.T. (1979) On the non-parallel flow stability of the Blasius boundary layer Proc. R. Soc. Lond. A 36691

Stokes, G.G. (1855) On the effect of the internal friction of fluids on the motions of pendulums Cambr. Phil. Trans. IX 8

Taylor, G.I. (1923) Stability of a viscous liquid contained between two rotating cylinders Phils. Trans. R. Soc. Lond. A 223289 
Figure 1 Inviscid growth rate curve

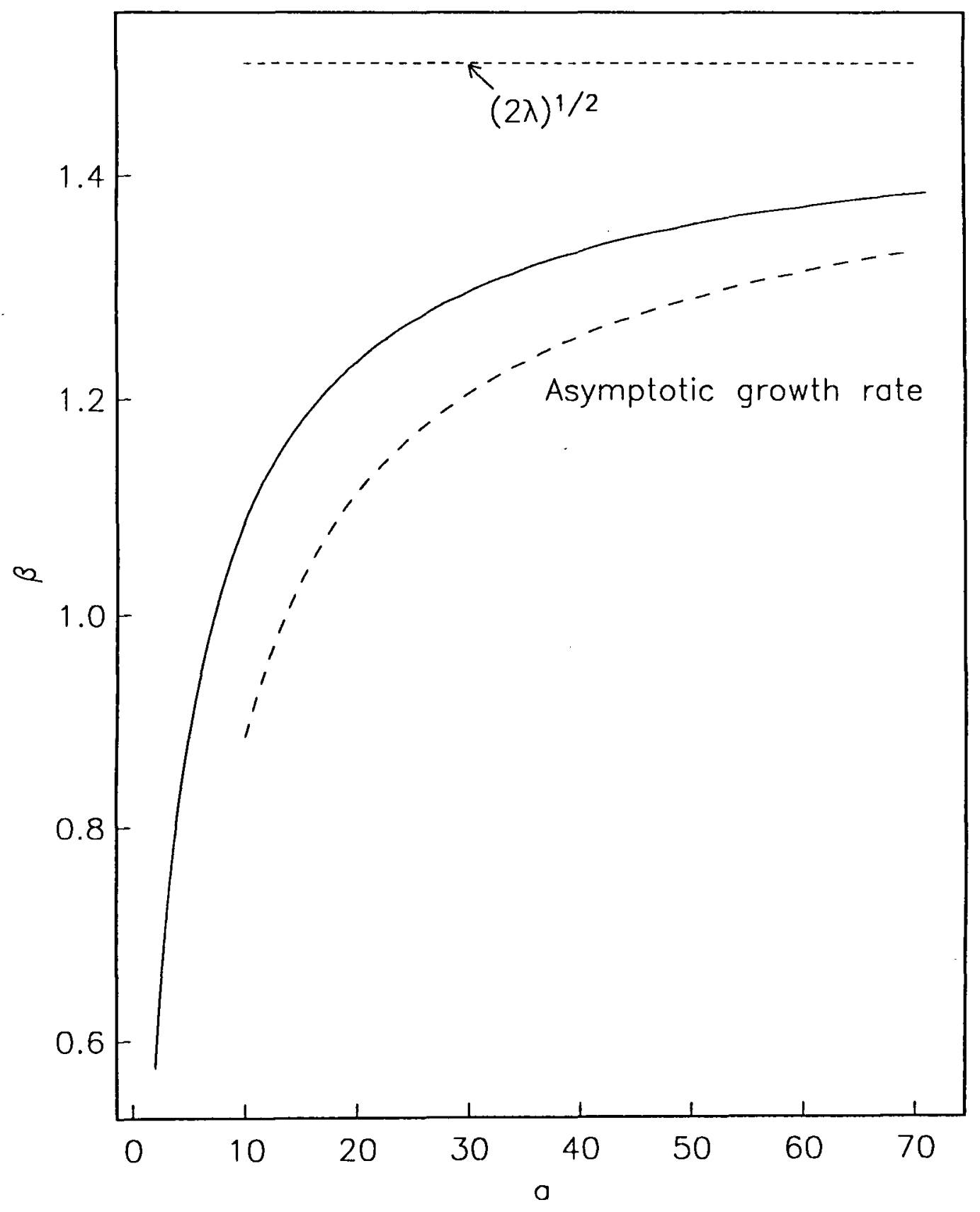

Figure 1: Inviscid growth rate curve, including asymptotic inviscid growth rate and the righthand branch growth rate value. 
Figure 2 The neutral curve

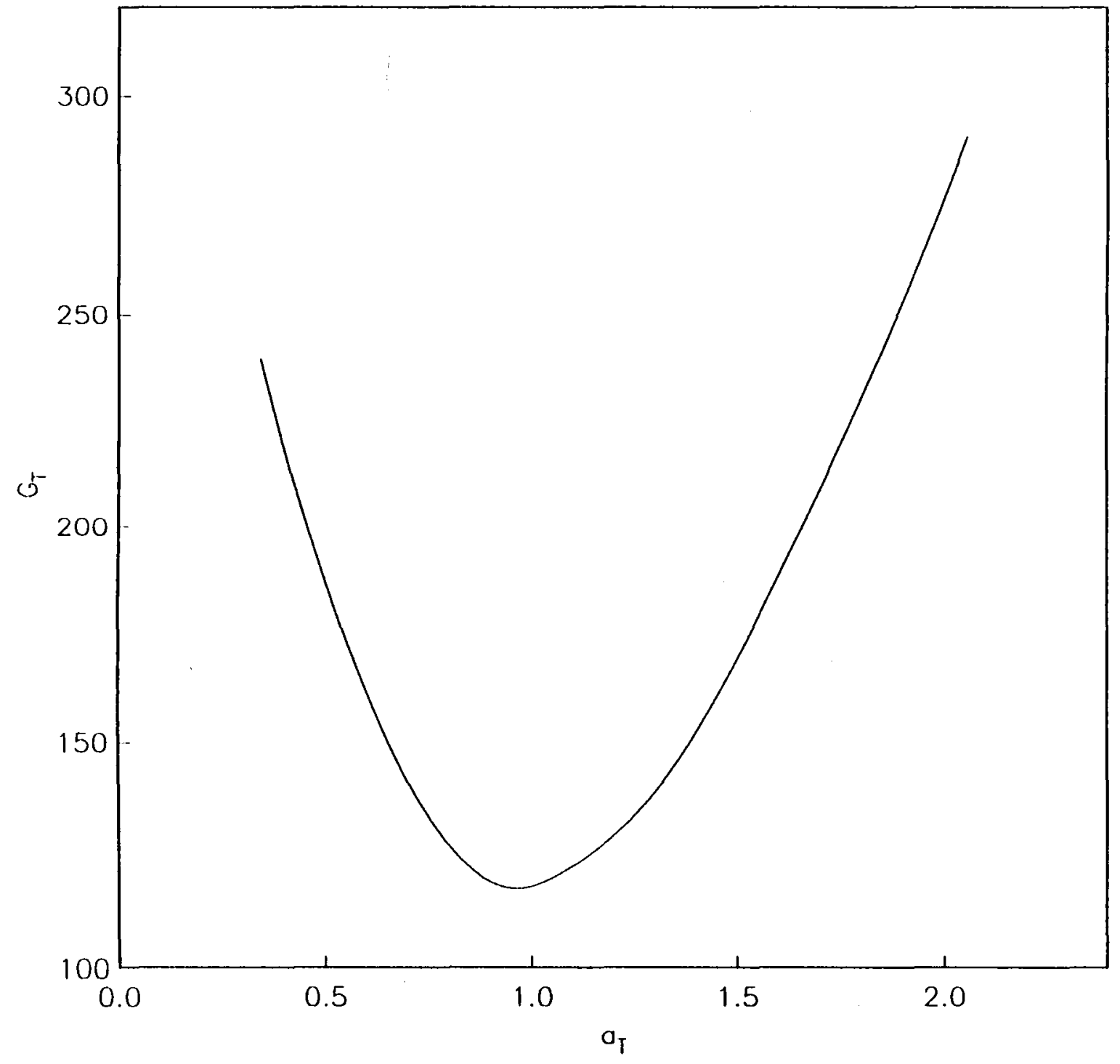

Figure 2: Neutral curve of local Görtler number $G_{T}$ against local wavenumber $a_{T}$, for a form of hump scaled on boundary layer thickness. 


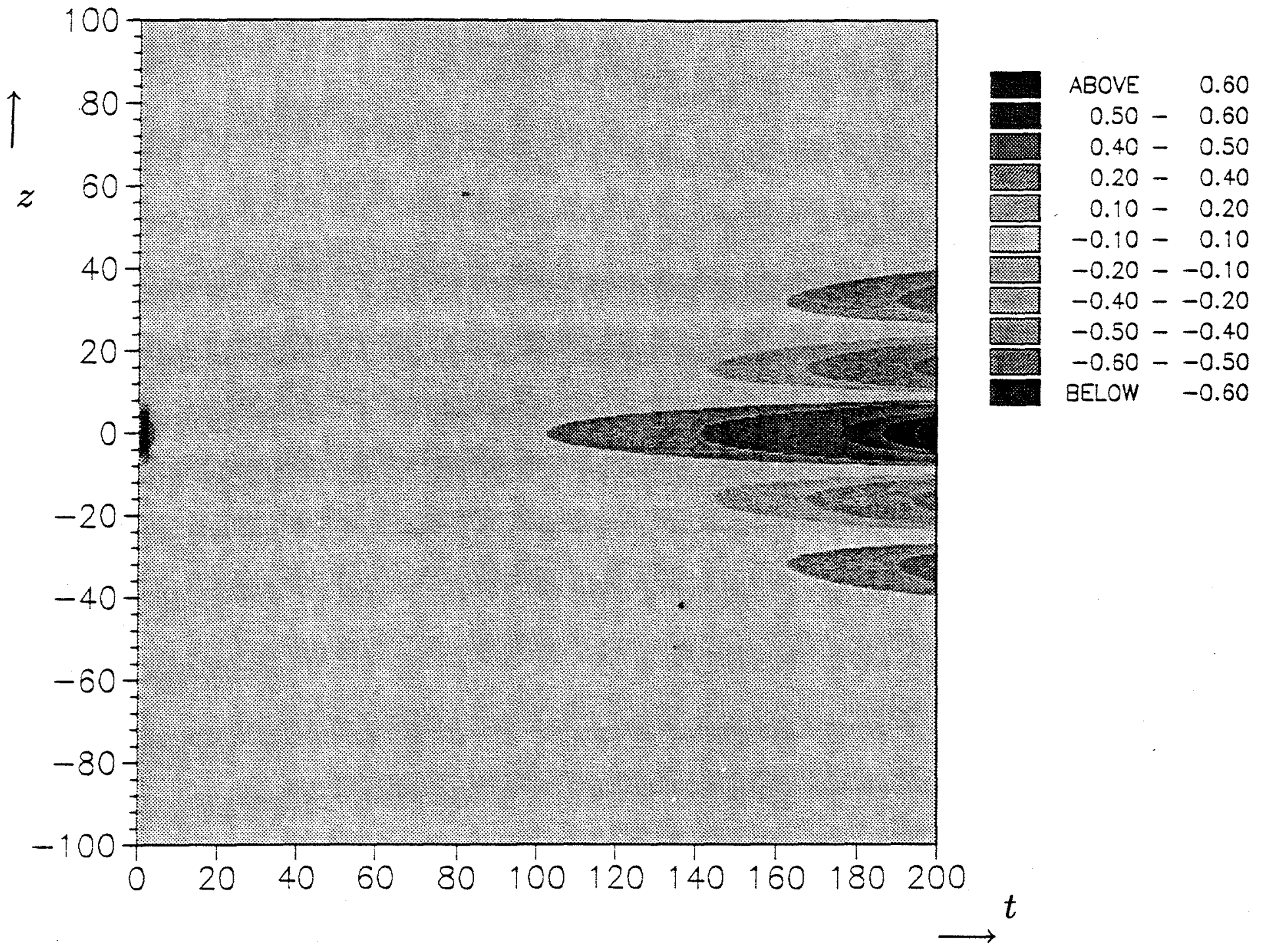

Figure 3: Contours of the maximum of the $V$ component of the vortex evolving with time, for a symmetric hump. 


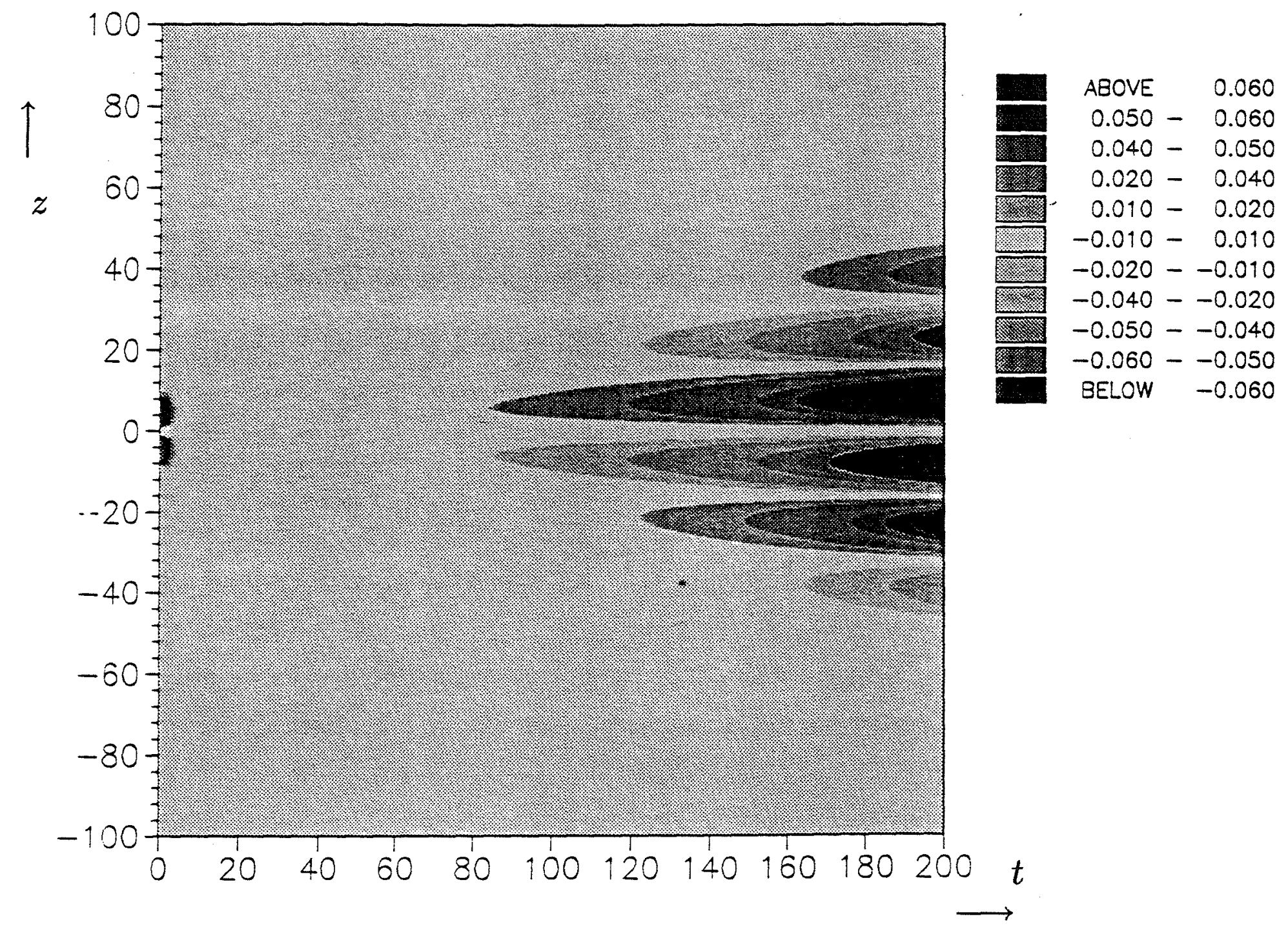

Figure 4: Contours of the maximum of the $V$ component of the vortex evolving with time, for an anti-symmetric hump. 


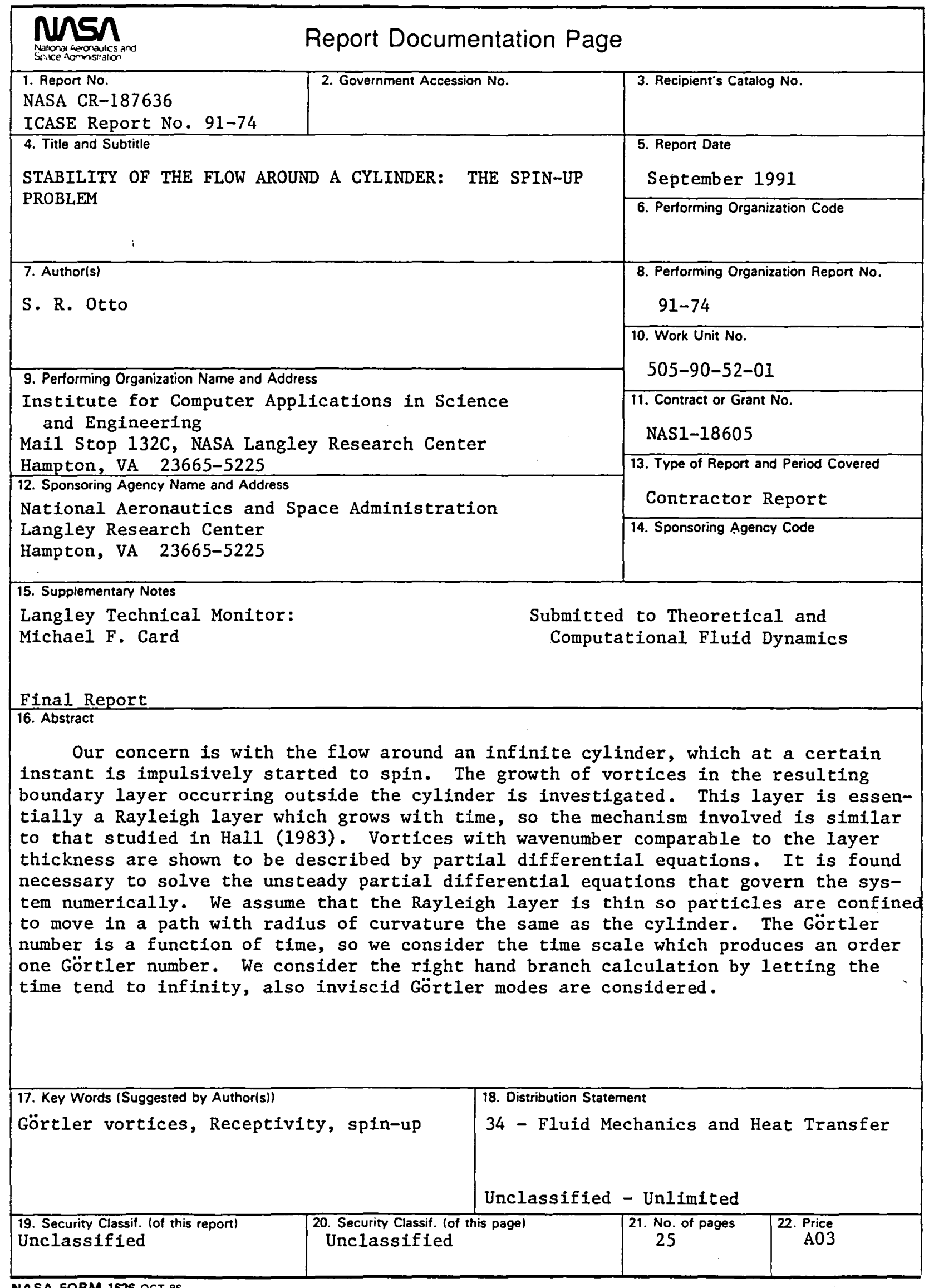


End of Document 